\title{
Ejercicios de léxico como material complementario para la traducción jurídica francés - español
}

\author{
MontserRat Cunillera Domènech \\ Universitat Pompeu Fabra \\ montserrat.cunillera@upf.edu
}

Recibido: 8 de febrero de 2011

Aceptado: 29 de febrero de 2012

\section{RESUMEN}

El presente trabajo propone una reflexión sobre el interés de elaborar y utilizar ejercicios de léxico como instrumento complementario para la enseñanza de la traducción jurídica. En esta propuesta los ejercicios se clasifican en dos grandes categorías según el momento en que se propongan a los estudiantes, antes o después de la traducción del texto, y según los objetivos, ya sea preparar la traducción del texto o bien revisar unidades léxicas previamente estudiadas. Su utilidad se pone de manifiesto en un doble sentido: contribuyen a enriquecer el bagaje léxico y cultural del estudiante en ambas lenguas, y a desarrollar habilidades cognitivas como la deducción y la reformulación, necesarias para llevar a cabo no sólo una traducción jurídica sino cualquier tipo de traducción.

Palabras clave: ejercicios de léxico, traducción jurídica, francés-español, deducción, reformulación.

Vocabulary exercises as complementary material for the French - Spanish legal translation

\begin{abstract}
This paper proposes a reflection on the interest to design and to use vocabulary exercises as complementary material for the teaching of legal translation. In this proposal exercises are classified in two main categories: according to the moment when they are suggested to the students, before or after translating the text, and according to the objectives, either to prepare the translation of the text or to review previous studied lexical/vocabulary units. Its usefulness is shown in a double sense: first of all, they contribute to enrich the lexical and cultural knowledge of the student in both languages, and secondly to develop cognitive abilities such as the deduction and the reformulation, both of them necessary to carry out not only a legal translation but any type of translation.
\end{abstract}


Keywords: vocabulary exercises, legal translation, French-Spanish, deduction, reformulation.

Sumario: 1. Introducción. 2. Traducción jurídica y técnicas traductoras. 3. Contexto académico y ejercicios en contexto. 4. Ejercicios de léxico. 4.1. Primera categoría: "pre-traducción”. 4.2. Segunda categoría: "post-traducción”. 5. Conclusiones. Referencias bibliográficas.

\section{Introducción}

En la traducción jurídica, el léxico no es el único pero sí uno de los elementos lingüísticos que mayores dificultades plantea. Cuando el estudiante de traducción se enfrenta a un texto jurídico, independientemente del género y del tema de que trata, topa con un léxico altamente complejo, no tanto por designar conceptos especializados sino por ser reflejo directo de una sociedad y una cultura concretas. A la complejidad de la materia se suma la escasez de manuales didácticos específicos para este tipo de traducción ${ }^{1}$, de manera que a menudo el docente debe elaborar él mismo sus propios materiales para ayudar a sus estudiantes a avanzar en tan ardua tarea. En este contexto marcado por el contraste entre necesidad y escasez, se inscribe pues la propuesta de ejercicios que expondré en las siguientes páginas. Se trata de ejercicios que focalizan la atención en el estudio del léxico y que pretenden contribuir, por un lado, a enriquecer el bagaje lingüístico, conceptual y cultural de los estudiantes en el campo del derecho y, por otro lado, a desarrollar determinadas habilidades cognitivas, como la deducción o la reformulación, necesarias para la traducción en general y, por tanto, también para la traducción jurídica.

\section{Traducción jurídica y técnicas traductoras}

La especificidad de la traducción jurídica radica en el uso de una lengua de especialidad (Duarte y Martínez 1995; Alcaraz y Hughes 2002), con un léxico y unas estructuras sintácticas y textuales muy particulares. Este tipo de traducción coincide con la traducción de otros lenguajes de especialidad como, por ejemplo, el de la ciencia y la tecnología, en el sentido de que en estos campos también es fundamental el conocimiento de un ámbito temático concreto. Ahora bien, se diferencia de ellos por su carácter marcadamente social y cultural.

El lenguaje jurídico, a diferencia del lenguaje de la ciencia o la tecnología, no remite a referentes extralingüísticos uniformes y universales, compartidos por diferentes sociedades, sino a referentes que derivan de las relaciones humanas, las cuales a su vez varían de una cultura a otra. El lenguaje jurídico refleja, pues, la complejidad de una sociedad, con su organización política, económica y judicial, cuyas

\footnotetext{
1 Para la traducción jurídica inglés-español afortunadamente contamos con obras interesantes como las de
} A. Borja (2000 y 2007), cuyos contenidos y propuestas son extrapolables a otras combinatorias lingüísticas. 
instituciones y vocabulario dependen directamente de una herencia cultural determinada y de su visión del mundo. De ahí que sea difícil que los órganos, instituciones o figuras jurídicas coincidan completamente en dos culturas. Por poner solo un ejemplo, sabemos que la Cour de Cassation y el Tribunal Supremo, pese a ser los tribunales supremos de Francia y España respectivamente, no son del todo equivalentes ya que no cumplen exactamente las mismas funciones ni poseen las mismas competencias. La complejidad de la traducción jurídica se hace más evidente cuando, además de transferir el texto jurídico de una cultura a otra, se ha de pasar de un sistema jurídico a otro, y todavía más si los sistemas jurídicos pertenecen a distintas familias de derecho. En el presente caso, puesto que la combinatoria lingüística de traducción es francés y español, nos encontramos ante una sola familia de derecho, dos culturas y dos sistemas jurídicos.

El léxico del derecho puede designar grosso modo dos tipos de referentes extralingüísticos: por un lado, instituciones y órganos jurisdiccionales y, por otro, nociones o conceptos jurídicos. Cuando los dos tipos de referentes no coinciden plenamente en las culturas confrontadas, se podrá optar entre diferentes técnicas traductoras $^{2}$ en función de ciertos parámetros derivados del encargo de traducción como la finalidad y los destinatarios a quienes vaya dirigido el nuevo texto, de acuerdo con el modelo funcionalista o la teoría del skopos (Vermeer 1996). Así, atendiendo a estos criterios, Cour de cassation podrá traducirse, por ejemplo, de forma literal (Tribunal de Casación), por su equivalente funcional (Tribunal Supremo francés) o por un préstamo (Cour de cassation). De forma análoga, los conceptos jurídicos crime, délit y contravention podrán traducirse de forma literal (crimen, delito, falta), por sus equivalentes funcionales (delito grave, delito menos grave, falta) o por los correspondientes préstamos, etc.

A pesar de que la finalidad de la traducción condiciona la elección de una determinada técnica traductora, en muchos casos las características mismas del vocablo jurídico original, ya sea por su contenido semántico o por su forma lingüística, inciden también en dicha elección. Así, en ocasiones, es preferible la traducción literal, por ejemplo, cuando no existe una equivalencia plena entre el término de la lengua original (LO) y el de la lengua meta (LM), como sucede entre Tribunal de grande instance y Tribunal de primera instancia o entre délits y delitos menos graves, o cuando no existe la misma figura en la cultura de llegada ni un equivalente funcional como, por ejemplo, la pena privativa de libertad denominada détention criminelle, que existe en el sistema jurídico francés pero no en el español. Y, en cambio, en otros casos, se descartará la traducción literal porque resulta engañosa y equívoca; por ejemplo, cuando los campos semánticos difieren y pueden crearse contrasentidos o falsos amigos (así, la détention provisoire corresponde a la prisión provisional y no a la detención), o bien cuando la traducción literal no crea sentido en la LM, lo que sucedería si se tradujeran de forma literal unidades como cour d'assises, magistrats du siège o garde des sceaux. En consecuencia, si el léxico jurídico se

\footnotetext{
2 Técnica traductora entendida como "procedimiento verbal concreto, visible en el resultado de la traducción, para conseguir equivalencias traductoras" (Hurtado 2001: 256-257).
} 
revela complejo y plantea dificultades en el momento de elegir una técnica traductora, será útil un trabajo de focalización, previo o ulterior a la traducción propiamente dicha del texto, que puede conseguirse mediante distintos tipos de ejercicios.

\section{Contexto académico y ejercicios en contexto}

Los ejercicios que presentaré en el siguiente apartado fueron diseñados en el marco de las asignaturas de traducción especializada jurídico-económica de francés (TEJE) de la antigua licenciatura en Traducción e Interpretación, cuyos objetivos generales eran ${ }^{3}$ :

- consolidar los conceptos fundamentales de las disciplinas de la economía y el derecho que los estudiantes han ido adquiriendo a fin de facilitarles la ubicación, la comprensión y la reformulación de los textos pertenecientes a estos ámbitos;

- potenciar la capacidad analítica de los alumnos en la clasificación de textos jurídico-económicos, en la detección y resolución de problemas traductológicos, en la definición de los objetivos que debe conseguir cada traducción y en los itinerarios de búsqueda más eficientes y eficaces en cada caso;

- dotar a los alumnos de las habilidades necesarias para poder realizar traducciones jurídico-económicas sobre cualquier tema y, a la vez, para ser capaces de ampliar sus conocimientos a lo largo de su vida profesional.

Los ejercicios de léxico se presentan como un instrumento metodológico susceptible de contribuir a la consecución de algunos de estos objetivos, concretamente la adquisición y consolidación de conceptos jurídicos y la ampliación de la competencia comunicativa y traductológica de los estudiantes. Estos ejercicios deben enmarcarse en una unidad didáctica más amplia basada en el análisis y la traducción de un texto jurídico. Es decir, los ejercicios de léxico permiten abordar un objetivo puntual, la competencia léxica y cultural, pero este cometido se inserta siempre en un objetivo último más general, la traducción de un texto jurídico. Por ello el léxico que se trabajará en dichos ejercicios se mostrará siempre contextualizado, es decir, acompañado del texto del que se haya extraído (el texto objeto de traducción).

En este caso, los ejercicios de léxico se basan en un texto jurisprudencial: la Sentencia $\mathrm{n}^{\circ} 2414$ de 30 de abril de 2002 del Tribunal de Casación francés, sobre un recurso basado en la presunta vulneración de determinadas normas procesales por parte del Tribunal de Apelación de Colmar (cf. texto íntegro en anexo). Así pues, el estudiante se enfrentará al lenguaje de los juristas (en oposición al lenguaje de las

3 Estos son los objetivos que recogía el Programa marco de las asignaturas de traducción especializada jurídico-económica de la licenciatura en Traducción e Interpretación de la UPF, diseñado por la profesora Lisa Gilbert (junio de 2002). 
leyes ${ }^{4}$ ) y a un texto perteneciente al ámbito del derecho procesal penal. Es evidente que un texto debe ir siempre acompañado de un hipotético encargo de traducción que podrá condicionar la elección de algunas de las técnicas traductoras. Sin embargo, en este trabajo, lo que interesa no es la traducción misma del texto sino ejercicios que contribuyan a la consecución de los objetivos mencionados anteriormente; por consiguiente, se prescindirá de la explicitación del encargo.

\section{Ejercicios de léxico}

Los ejercicios de léxico constituyen un instrumento metodológico que complementa el ejercicio de la traducción propiamente dicha y pueden proponerse antes o después de la traducción según los objetivos previstos. Para mi propuesta mostraré dos posibles planteamientos que obedecen a dos finalidades distintas: en el primer caso, que denominaré "pre-traducción", se trata de facilitar o preparar la operación traductora y por ello los ejercicios serán previos a la traducción; en el segundo caso o "post-traducción", se pretende revisar unidades léxicas ya estudiadas, por lo que los ejercicios serán posteriores a la traducción. Cada planteamiento permite diversos modelos de ejercicios que ilustraré con ejemplos concretos para mostrar más claramente en qué consisten así como las habilidades lingüísticas o cognitivas que permiten desarrollar. El orden de los ejercicios no está relacionado con el grado de dificultad de los mismos ni pretende reflejar ningún tipo de progresión en el aprendizaje.

\subsection{Primera categoría: "pre-traducción"}

En primer lugar, se proporciona un texto jurídico al estudiante, que deberá leer y comprender de forma global. En esta primera aproximación, el estudiante dejará de lado las unidades léxicas que desconozca (si lo desea, las podrá marcar para luego identificarlas más rápidamente pero no buscar su significado), y deberá intentar comprender únicamente el sentido global del texto. En segundo lugar, se propondrán los ejercicios de léxico, tras los cuales se procederá a la traducción propiamente dicha del texto.

Esta primera categoría está formada por cuatro modelos de ejercicios. Los tres primeros son de comprensión lectora y de producción léxica, más activos o pasivos según si el objetivo consiste en encontrar un término o bien seleccionar uno de los propuestos por el docente. Estos ejercicios permiten que el estudiante descubra léxico nuevo de un determinado campo temático, tanto en la LO como en la LM,

4 Cf. Iturralde Sesma 1989: 30.

Algunos autores distinguen entre lenguaje legal, que se emplea para la redacción de normas, y el de los juristas, que es el que emplean los juristas cuando hablan, aplican o interpretan dichas normas (Duarte y Martínez 1995: 43). 
ampliando así su competencia léxica, conceptual y cultural. El último ejercicio, de traducción directa, tiene como objetivo profundizar en el conocimiento de unidades con contornos semánticos difusos, sin equivalentes directos en la LM, y potenciar técnicas traductoras como, por ejemplo, la reformulación. Estos ejercicios se realizarán siempre en contexto, es decir, el estudiante deberá tener presente el texto de donde se ha extraído el léxico.

\section{Ejercicio 1: producción léxica}

En este ejercicio se propone la definición lexicográfica de un término concreto, extraída de un diccionario monolingüe, para que el estudiante llegue a deducir el término a que hace referencia. Se basa en un procedimiento de carácter onomasiológico ya que se parte de la definición para llegar al signo lingüístico. Es importante potenciar esta estrategia de carácter deductivo porque se utiliza en una de las etapas de la operación traductora: cuando el estudiante, a partir de la definición de un término en un diccionario monolingüe, debe ser capaz de hallar el término en la LM. Este primer ejercicio permite diversas modalidades: se puede partir de la definición en LO para llegar al término en LO o bien de la definición en LM para llegar al término en LM (aquí pueden trabajarse elementos culturales como instituciones y órganos jurisdiccionales). Por ejemplo:

- Représentant du ministère public et chef du Parquet près du tribunal de grande instance. (De Fontette 1988: 99)

FR:

- Es la privación temporal de la libertad de una persona ante la sospecha de que sea responsable de una infracción penal [...] Tal medida en principio sólo cabe respecto de los delitos, no de las faltas [...] Su duración no podrá exceder del plazo de setenta y dos horas [...] (Fernández Martínez 2002: 286)

CAST:

O incluso, se puede partir de la definición en LO para llegar tanto al término en LO como al término en LM. Aquí solo se pueden trabajar conceptos que posean el mismo contenido jurídico en ambas lenguas. En esta modalidad, el estudiante debe llevar a cabo un proceso deductivo doble a partir de la definición proporcionada, que ha de culminar en una actividad de producción también doble; es decir, debe llegar a la formulación adecuada en la LO y en la LM.

- Juge qui s'occupe d'une phase du procès pénal qui est facultative en matière de délits, obligatoire à deux degrés (...) pour les crimes, et qui permet d'établir les circonstances et les conséquences de l'infraction et de décider si les charges sont suffisantes pour qu'une juridiction de jugement soit saisie. (Guillien y Vincent 1995: 321)

CAST:

FR:

Ejercicio 2: selección léxica

Este ejercicio se basa en un procedimiento onomasiológico similar al del ejercicio anterior: también se parte de la definición para llegar al término. Sin embargo, 
en este caso se proporcionan dos o tres términos para que el estudiante escoja uno de ellos como solución correcta. El alumno debe llevar a cabo un proceso deductivo (entender la definición lexicográfica en francés), paralelamente a una actividad cognitiva de identificación y selección (escoger uno de los términos propuestos). El hecho de disponer de distintos términos sabiendo que uno de ellos es correcto facilita la operación cognitiva, pero también puede exigir un trabajo adicional puesto que el estudiante se verá obligado a buscar el significado de los términos que no conozca a ciencia cierta para asegurarse de que su elección es la correcta. Así, a partir de la definición en la LO, el estudiante debe escoger un término de los tres propuestos en la LO. Por ejemplo:

- Représentant du ministère public et chef du Parquet près du tribunal de grande instance. (De Fontette 1988: 99)

\begin{tabular}{|lll|}
\hline procureur général & rapporteur procureur de la République \\
\hline
\end{tabular}

El mismo tipo de ejercicio podría realizarse también con la LM:

- Es la parte dispositiva de la resolución judicial y por tanto la principal, en cuanto que es a dicha parte a la que se vinculan los efectos de la cosa juzgada. (Fernández Martínez 2002: 345)

fallo disposición providencia

Ejercicio 3: asociación de términos y definiciones

En este tercer ejercicio se presentan una serie de términos y definiciones que el estudiante debe relacionar, siendo posibles como mínimo dos modalidades. En la primera modalidad, se incluye una lista de términos tomados del texto que será objeto de traducción y una lista de definiciones lexicográficas en la LO extraídas de un diccionario monolingüe. Los términos y las definiciones están desordenados y el estudiante debe asociar cada término con su definición. Esta modalidad está basada en un procedimiento de tipo semasiológico puesto que el estudiante debe inferir cuál es la definición de cada término francés (del signo lingüístico debe llegar al contenido semántico). Por ejemplo: 


\begin{tabular}{|l|l|}
\hline TÉRMINOS & DEFINICIONES \\
\hline & $\begin{array}{l}\text { a) } \begin{array}{l}\text { Mesure d'incarcération d'un inculpé pendant } \\
\text { l'information judiciaire, ou d'un prévenu dans le } \\
\text { cadre de la comparution immédiate. (...) (Guillien } \\
\text { y Vincent 1995: 204) }\end{array} \\
\text { 1) chambre criminelle }\end{array}$ \\
$\begin{array}{l}\text { bétention provisoire } \\
\text { 3) pourvoi } \\
\text { pourvois en cassation ou en révision en matière } \\
\text { pénale. (De Fontette 1988: 23) }\end{array}$ \\
$\begin{array}{l}\text { c) Recours extraordinaire contre une décision rendue } \\
\text { en dernier ressort et porté en général devant la Cour } \\
\text { de cassation. (De Fontette 1988: 96) }\end{array}$ \\
\hline
\end{tabular}

La segunda modalidad contiene, además de las dos listas anteriores, otra lista con los términos equivalentes en la LM. En este caso, el estudiante deberá enlazar cada una de las primeras combinaciones con el término o expresión de la última lista, teniendo siempre en cuenta el texto en que aparecen. Los términos propuestos en LM se mostrarán preferiblemente mezclados con distractores a fin de dificultar la tarea de selección. Por ejemplo, para cada término en francés se pueden proponer dos posibles equivalencias léxicas en castellano, siendo correcta sólo una de ellas. Esta modalidad está basada, por una parte, en un procedimiento de tipo semasiológico (como la anterior) y, por otra parte, en una operación onomasiológica puesto que también se debe escoger, tras comprender la definición, un signo lingüístico en LM de los propuestos en la tercera columna.

\begin{tabular}{|c|c|c|}
\hline $\begin{array}{l}\text { TÉRMINOS EN } \\
\text { FRANCÉS }\end{array}$ & DEFINICIONES & $\begin{array}{l}\text { TÉRMINOS EN } \\
\text { CASTELLANO }\end{array}$ \\
\hline 1) chambre criminelle & $\begin{array}{l}\text { a) Mesure d'incarcération d'un } \\
\text { inculpé pendant l'information } \\
\text { judiciaire, ou d'un prévenu dans } \\
\text { le cadre de la comparution } \\
\text { immédiate. (Guillien y Vincent } \\
\text { 1995: 204) }\end{array}$ & $\begin{array}{l}\text { detención } \\
\text { sala de lo penal }\end{array}$ \\
\hline 2) détention provisoire & $\begin{array}{l}\text { b) }[. . .] \text { à la Cour de cassation statue } \\
\text { sur les pourvois en cassation } \\
\text { ou en révision en matière pénale. } \\
\text { (De Fontette 1988: } 23 \text { ) }\end{array}$ & $\begin{array}{l}\text { recurso de casación } \\
\text { recurso de apelación }\end{array}$ \\
\hline 3) pourvoi & $\begin{array}{l}\text { c) Recours extraordinaire contre une } \\
\text { décision rendue en dernier ressort } \\
\text { et porté en général devant la Cour } \\
\text { de cassation. (De Fontette 1988: } \\
96 \text { ) }\end{array}$ & $\begin{array}{l}\text { prisión preventiva } \\
\text { sala de instrucción }\end{array}$ \\
\hline
\end{tabular}

Ejercicio 4: traducción de lexemas con contornos semánticos vagos 
El cuarto ejercicio aborda el estudio del léxico desde una perspectiva distinta. En este caso se trata de trabajar con unidades léxicas que poseen unos contornos semánticos vagos y poco precisos, por lo que carecen de un equivalente directo y unívoco en la LM. El estudiante deberá producir una expresión o formulación que transfiera el mismo valor semántico que la unidad original. Gracias al procedimiento que este ejercicio obliga a seguir, se puede conseguir un doble cometido: por una parte, profundizar en el conocimiento de una determinada unidad léxica - ya que el ejercicio enfrenta al estudiante a dicha unidad haciéndole descubrir sus valores semánticos y las construcciones sintácticas en que interviene- y, por otra parte, desarrollar la estrategia traductora de la reformulación -puesto que el estudiante, ante la ausencia de equivalentes directos en la LM, debe recurrir a distintos tipos de fórmulas para expresar el mismo sentido que el texto de partida-. Esta habilidad lingüística y cognitiva es la que necesita adquirir el estudiante para enfrentarse a la traducción de numerosos términos jurídicos.

El ejercicio estará compuesto por distintas ocurrencias de la unidad léxica que se desee trabajar, extraídas siempre del texto que debe ser objeto de traducción. Para ejemplificar este tipo de ejercicio he escogido una unidad léxica muy frecuente en el ámbito procesal francés: el verbo saisir. Esta unidad se caracteriza además por la posibilidad de formar parte de distintas construcciones sintácticas que inciden en la cristalización de uno de sus sentidos $\mathrm{y}$, por tanto, determinan las posibles soluciones traductoras. Como requisito previo, se pedirá a los estudiantes que se documenten y reflexionen sobre los valores de dicha unidad. Para ello deberán, en primer lugar, buscar su significado en un diccionario monolingüe a fin de conocer sus distintas acepciones, usos y construcciones sintácticas y, en segundo lugar, consultar un diccionario bilingüe de especialidad para valorar las equivalencias consignadas y decidir si es posible escoger alguna de ellas para traducir las ocurrencias que contiene el ejercicio o si, al contrario, es preferible crear una nueva formulación.

- Attendu qu'il résulte des dispositions précitées, que, lorsque le juge d'instruction, (a) saisi de réquisitions aux fins de placement en détention provisoire ou sous contrôle judiciaire d'une personne, ne la met pas en examen et ne rend pas d'ordonnance, le procureur de la République peut (b) saisir directement la chambre de l'instruction (...) (Arrêt $\mathrm{n}^{\circ} 241230$ avril 2002).

a)

b)

\subsection{Segunda categoria: "post-traducción"}

Los ejercicios de esta categoría tienen como objetivo principal revisar el léxico que ya ha sido objeto de estudio previamente; por lo tanto, se propondrán una vez traducido el texto. En una sesión posterior a la corrección de la traducción, se proporcionarán ejercicios para activar o movilizar determinados conocimientos lingüísticos y culturales. De esta forma, se comprueba si el estudiante ha asimilado dichas unidades y si es capaz de producirlas de nuevo. En esta categoría presentaré 
dos modelos de ejercicios: el primero de traducción directa y el segundo de traducción inversa.

Ejercicio 1: traducción de lexemas con contornos semánticos vagos

Este ejercicio se basa en el mismo mecanismo que el ejercicio 4 y pretende objetivos similares; es decir, se propone la traducción de una unidad léxica sin equivalente directo y unívoco con el fin de potenciar la competencia de la producción en la LM y la técnica traductora de la reformulación. Ahora bien, el planteamiento es distinto ya que se propone no antes sino después de la traducción del texto y el objetivo concreto consiste en activar unidades ya estudiadas para traducirlas o utilizarlas en nuevos contextos. Por ejemplo, si retomamos el verbo saisir del ejercicio 4, ahora se tratará de presentar más ocurrencias de esta unidad, extraídas de otros textos jurídicos, con el objetivo de poder ofrecer un abanico más amplio de contextos en los que deba recuperarse mediante formulaciones distintas. El contexto en que se encuentre cada ocurrencia de la unidad objeto de estudio será imprescindible para aprehender su sentido y facilitar la producción de un equivalente en la LM.

- Pourront néanmoins les juges, saisis de la cause et statuant sur le fond, prononcer la suppression des discours injurieux, outrageants ou diffamatoires, et condamner qui il appartiendra à des dommages-intérêts. (Art. 41, Loi sur la liberté de la presse)

- Depuis sa création en 1952 jusqu'à nos jours, la Cour de justice a été saisie de plus de 8.600 affaires. Le chiffre de 200 nouvelles affaires par an a été atteint dès 1978 et, en 1985, le chiffre de 400 affaires par an a été dépassé. ("La procédure des recours directs devant la Cour de justice", http://curia.eu.int/fr/index.htm)

Por último, y para continuar con la reflexión gramatical sobre saisir iniciada en el ejercicio 4, se podrá pedir a los estudiantes que, a partir de la información obtenida de las obras lexicográficas consultadas y de las traducciones de saisir propuestas en el ejercicio 4 y en el presente, elabore una tabla con las principales construcciones sintácticas en que interviene el verbo saisir y las posibles equivalencias en cada caso.

Ejercicio 2: aproximación a la traducción inversa

La traducción inversa comporta siempre mayor dificultad por razones evidentes, por lo que, antes de enfrentar al estudiante a la traducción de un texto jurídico completo a su lengua no materna, puede ser útil llevar a cabo una aproximación a este tipo de traducción mediante la realización de ejercicios de léxico más elementales como, por ejemplo, un ejercicio de huecos. Los objetivos en este caso consisten en potenciar la competencia léxica del estudiante en su lengua no materna, y revisar unidades o construcciones lingüísticas abordadas en la traducción directa. 
Este modelo de ejercicio debe estar compuesto por un conjunto de fragmentos de textos jurídicos en español acompañados de sus respectivas traducciones al francés, las cuales deben contener espacios en blanco para que el estudiante los complete con las unidades que faltan. Al igual que en los ejercicios anteriores, se puede focalizar la atención en unidades que remitan a elementos culturales y a contenidos jurídicos específicos, o bien en unidades léxicas y construcciones sintácticas que sean características del lenguaje jurídico francés con contornos semánticos vagos y poco precisos. Asimismo, puede ser útil proponer su realización después de haber estudiado los valores semánticos de una determinada unidad léxica en francés y sus posibles traducciones al castellano. Por ejemplo, tras haber realizado el ejercicio 4 de la primera categoría y el ejercicio 1 de la segunda categoría, se propondrá un ejercicio de huecos en el que se pueda utilizar la unidad saisir para traducir un elemento léxico del texto español. Si, además, el texto castellano presenta otros elementos léxicos ya comentados y analizados en clase a partir de la traducción directa, puede resultar interesante la posibilidad de retomarlos desde la perspectiva de la traducción inversa. Con ese propósito, se dejarán nuevos huecos en el texto de llegada, y se destacarán en negrita las unidades del texto de partida que deban traducirse. De esta forma, se puede comprobar si el estudiante ha asimilado las unidades léxicas ya estudiadas y si es capaz de producirlas en su lengua extranjera. ${ }^{5}$

1a) Los conflictos negativos [conflictos de competencia entre el Estado y las comunidades autónomas]

$\mathrm{Si}$, a diferencia del caso de los conflictos positivos, ninguna instancia se considera competente para llevar a cabo una determinada actuación solicitada por una persona física o jurídica, esta persona [...] puede acudir al Tribunal Constitucional para que sea éste el que decida a quien corresponde dicha competencia. (Gerpe 2002: 69)

1b) Les conflits négatifs [conflits de compétence entre l'Etat et les communautés autonomes]

Si, contrairement aux conflits positifs, aucune instance ne se considère compétente pour agir d'une certaine façon à la demande d'une personne. cette personne [...] ........... le ........... afin que celui-ci décide à qui revient la compétence. ${ }^{6}$

2a) La cámara [baja], a través de la solicitud que cursa el Tribunal Supremo [...], ha de autorizar o no de manera motivada y fundada el inicio de las acciones judiciales contra los parlamentarios. Se puede recurrir la decisión de las cámaras ante el Tribunal Constitucional. (Gerpe 2002: 43)

\footnotetext{
${ }^{5}$ Los dos fragmentos en castellano de este ejercicio son a su vez una traducción del catalán, que ha realizado la autora del artículo con motivo del mismo.

${ }^{6}$ Posible solución: ...à la demande d'une personne PHYSIQUE OU MORALE, cette personne [...] PEUT SAISIR le TRIBUNAL CONSTITUTIONNEL (/CONSEIL CONSTITUTIONNEL) afin que celui-ci décide à qui revient la compétence.
} 
2b) La chambre, [...] doit autoriser ou non d'une façon motivée et fondée contre les parlamentaires. La décision des chambres devant le .............. ${ }^{7}$

\section{Conclusiones}

La presente propuesta ha pretendido reflexionar sobre la conveniencia de abordar la enseñanza de la traducción jurídica mediante ejercicios de léxico complementarios, poniendo de manifiesto algunas de sus ventajas. En primer lugar, contribuyen a enriquecer el bagaje léxico y cultural del estudiante en ambas lenguas y en distintos ámbitos temáticos del derecho. El estudio del léxico permite pasar de aspectos microtextuales a aspectos conceptuales, puesto que junto a una forma lingüística el estudiante descubre un determinado contenido jurídico, y a aspectos culturales, ya que a partir del léxico se abordan elementos propios de la cultura de partida y de la cultura de llegada. En segundo lugar, los ejercicios realizados en el aula permiten participar directa y espontáneamente a los estudiantes: entre otras cosas, exigen pensar con rapidez para entender la definición y encontrar el término al que se refiere. De este modo, al realizar tales actividades, se potencian habilidades cognitivas como la deducción y la reformulación, necesarias para llevar a cabo no sólo una traducción jurídica sino cualquier tipo de traducción. Así, mediante los ejercicios basados en un movimiento de deducción, el estudiante lleva a cabo una actividad cognitiva muy parecida a la que realiza cuando consulta la definición de un término de la $\mathrm{LO}$ en un diccionario monolingüe para hallar el término en la LM. Y en cuanto a los ejercicios basados en la reformulación, permiten desarrollar una técnica traductora útil para recuperar unidades léxicas sin equivalentes directos en la LM (como se ha visto con la unidad saisir).

Teniendo en cuenta que actualmente escasean los materiales y recursos para la enseñanza de este tipo de traducción, la publicación de las actividades que realizamos los docentes puede ayudar a colmar ciertas lagunas; resulta conveniente, pues, seguir ampliando y mejorando dichos instrumentos a partir de las necesidades existentes en este campo.

\section{Referencias bibliográficas}

Alcaraz Varó, E., Hughes, B., El Español Jurídico. Barcelona: Ariel Derecho 2002. Arrêt de la Cour de cassation n ${ }^{\circ}$ 2412, 30 avril 2002 [consulta en línea: http://www.courdecassation.fr/agenda/arrets/arrets/arretnav.htm; 02/02/2011]

Borja, A., El texto jurídico inglés y su traducción al español. Barcelona: Ariel 2000.

Borja, A., Estrategias, materiales y recursos para la traducción jurídica inglés-español.

\footnotetext{
7 Posible solución: La chambre, SAISIE D'UNE REQUETTE DE LA COUR SUPRÊME [...] doit autoriser ou non d'une façon motivée et fondée LA SAISINE DES POURSUITES contre les parlamentaires. La décision des chambres EST SUSCEPTIBLE D'APPEL devant le TC.
} 
Castelló: Universitat Jaume I 2007.

Duarte, C., Martínez, A., El Lenguaje Jurídico. Buenos Aires: A-Z Editora 1995.

Fernández Martínez, J.M., Diccionario jurídico. Navarra: Aranzadi 2002.

Fontette, F. De, Vocabulaire Juridique. Francia: PUF 1988.

Gerpe, M., Dret constitucional II. Barcelona: UOC 2002.

Guillien, R., Vincent, J., Termes Juridiques. Francia: Dalloz 1995.

Hurtado Albir, A., Traducción y traductología. Introducción a la traductología. Barcelona: Cátedra 2001.

Iturralde Sesma, V., Lenguaje Legal y Sistema Jurídico. Madrid: Tecnos 1989.

«La procédure des recours directs devant la Cour de justice» [consulta en línea: http://curia.eu.int/fr/index.htm; 10/01/2011]

Loi 29/07/1881 sur la liberté de la presse. Le Journal Officiel de la République Française [consulta en línea: www.legifrance.gouv.fr; 10/01/2011]

Vermeer, H. J., A skopos theory of translation. Heidelberg: TEXTconTEXT-Verlag 1996. 
Anexo

Texto francés en que se han basado los ejercicios de léxico ${ }^{8}$

\section{2-82.017}

Arrêt $n^{\circ} 2412$ du 30 avril 2002

Cour de cassation - Chambre criminelle

Cassation

Demandeur(s) à la cassation: Procureur général près la cour d'appel de Colmar

$\mathrm{Vu}$ l'ordonnance du président de la chambre criminelle, en date du 20 mars 2002, prescrivant l'examen immédiat du pourvoi;

$\mathrm{Vu}$ le mémoire produit et les mémoires en défense;

Sur le moyen unique de cassation, pris de la violation des articles 82 et 137-5 du Code de procédure pénale;

Vu l'article 82, alinéas 1, 4 et 5 du Code de procédure pénale;

Attendu qu'il résulte des dispositions précitées, que, lorsque le juge d'instruction, saisi de réquisitions aux fins de placement en détention provisoire ou sous contrôle judiciaire d'une personne, ne la met pas en examen et ne rend pas d'ordonnance, le procureur de la République peut saisir directement la chambre de l'instruction;

Attendu qu'il appert de l'arrêt attaqué et des pièces de la procédure que, le 17 janvier 2002, le procureur de la République a requis l'ouverture d'une information notamment contre X..., Y..., Z..., du chef de viols en réunion sur mineure de quinze ans; qu'il a également requis leur placement en détention provisoire; que le juge d'instruction ayant seulement procédé, le même jour, à l'audition des intéressés, en tant que témoins assistés, le procureur de la République, par requête du 22 janvier suivant, a saisi directement la chambre de l'instruction en vue de leur mise en examen et de leur placement en détention;

Attendu que, pour déclarer la requête irrecevable, les juges retiennent "qu'aucune disposition de la loi n'a prévu que le procureur de la République, lorsqu'il a ouvert une information contre personne dénommée et que cette personne n'a pas été mise en examen par le juge d'instruction qui lui a conféré le statut de témoin assisté, puisse former un recours devant la chambre de l'instruction";

Mais attendu qu'en prononçant ainsi, les juges ont méconnu le texte susvisé et le principe ci-dessus rappelé;

D'où il suit que la cassation est encourue;

PAR CES MOTIFS:

CASSE et ANNULE, en toutes ses dispositions l'arrêt précité de la chambre de l'instruction de la cour d'appel de Colmar, en date du 7 février 2002, et pour qu'il soit à nouveau jugé conformément à la loi;

\footnotetext{
${ }^{8}$ http://www.courdecassation.fr/agenda/arrets/arrets/arretnav.htm
} 
RENVOIE la cause et les parties devant la chambre de l'instruction de la cour d'appel de Nancy;

Président : M. Cotte

Rapporteur : Mme Karsenty, conseiller référendaire

Avocat général : $M$. Davenas

Avocat(s) : Me Le Prado, la SCP Bachellier et Potier de la Varde 